Check for updates

Cite this: RSC Adv., 2018, 8, 26271

Received 14th June 2018

Accepted 17th July 2018

DOI: 10.1039/c8ra05108a

rsc.li/rsc-advances

\section{Synthesis of an oxygen-linked germinal frustrated Lewis pair and its application in small molecule activation $\uparrow$}

\author{
Yiheng Wang, ${ }^{a}$ Zhen Hua Li (iD ${ }^{a}$ and Huadong Wang (D)*ab
}

Reaction of $\mathrm{Mes}_{2} \mathrm{P}(\mathrm{O}) \mathrm{Li}$ with $\left(\mathrm{C}_{6} \mathrm{~F}_{5}\right)_{2} \mathrm{BCl}$ gave access to an oxygen-linked germinal intramolecular frustrated Lewis pair $\mathrm{Mes}_{2} \mathrm{P}(\mathrm{O}) \mathrm{B}\left(\mathrm{C}_{6} \mathrm{~F}_{5}\right)_{2}$ (1). Compound 1 is stable at room temperature and only decomposes when heated to $90{ }^{\circ} \mathrm{C}$. NMR analysis and theoretical analysis revealed the frustrated nature between the boron and phosphorus centers. Compound 1 shows typical frustrated Lewis pair reactivity when treated with dihydrogen, carbon dioxide, alkyne and alkene. The reaction of 1 with isoprene resulted in selective formation of 3,4-phosphoryl/boryl addition product 8 .

\section{Introduction}

Ever since the groundbreaking discovery by Stephan et al. that an unquenched phosphine/borane pair can activate dihydrogen under ambient conditions, the concept of a "frustrated Lewis pair" (FLP) has been successfully applied in small-molecule activation, organic synthesis and catalysis. ${ }^{1}$ Mechanism investigations revealed that one of the most prominent features of FLP mediated reactions is that substrates are usually synergistically activated by both the Lewis acid and base centers. ${ }^{2}$ To enhance such a synergistic effect, the most often employed strategy is to pre-organize Lewis acid and base functionalities through a linker. ${ }^{3}$ Among a variety of intramolecular FLPs, germinal FLPs in which donor and acceptor sites are separated by one atom have attracted substantial attention because the proximity of their Lewis acid and base centers can facilitate their synergistic reactivities against small molecules. Furthermore, despite such proximity, intramolecular interaction between Lewis acid and base centers in germinal FLPs is largely excluded because of the high constrain of formation of a threemembered ring. Attempts to introduce frequently applied $-\mathrm{B}\left(\mathrm{C}_{6} \mathrm{~F}_{5}\right)_{2}$ and $-\mathrm{P} t \mathrm{Bu}_{2}$ functionalities to C1-linked FLPs were thwarted by facile intramolecular nucleophilic substitution of fluoride on the $\mathrm{C}_{6} \mathrm{~F}_{5}$ ring by the phosphine moiety $(\mathbf{I}) .{ }^{4}$ To circumvent this problem, Slootweg, Lammertsma and coworkers employed $-\mathrm{BPh}_{2}$ instead of $-\mathrm{B}\left(\mathrm{C}_{6} \mathrm{~F}_{5}\right)_{2}$ functionality and synthesized a $\mathrm{CH}_{2}$-bridged $t \mathrm{Bu}_{2} \mathrm{PCH}_{2} \mathrm{BPh}_{2}$ (II). ${ }^{5}$ It was

\footnotetext{
${ }^{a}$ Department of Chemistry and Shanghai Key Laboratory of Molecular Catalysis and Innovative Materials, Fudan University, Songhu Road 2005, Shanghai, 200438, China ${ }^{b}$ State Key Laboratory of Elemento-Organic Chemistry, Nankai University, Tianjin, 300071, China. E-mail: huadongwang@fudan.edu.cn

$\dagger$ Electronic supplementary information (ESI) available: [DETAILS]. CCDC 1843771-1843777 (2-8). For ESI and crystallographic data in CIF or other electronic format see DOI: 10.1039/c8ra05108a
}

discovered that this germinal FLP can activate $\mathrm{H}_{2}, \mathrm{CO}_{2}$ and other unsaturated substrates despite of the mild Lewis acidity of the boron center. The groups of Wagner ${ }^{6}$ and Erker ${ }^{7}$ also reported a number of methylene-bridged germinal FLPs employing $-\mathrm{BFxyl}_{2}(\mathrm{Fxyl}=3,5$-bis(trifluoromethyl)phenyl) as the Lewis acid center (III) or less nucleophilic $-\mathrm{P}\left(\mathrm{C}_{6} \mathrm{~F}_{5}\right)_{2}$ as the Lewis base center (IV). Besides of methylene linkers, alkylidene moiety was also employed as a linker for germinal FLPs. Uhl and coworkers prepared a series of alkylidene-bridged Al/P based FLPs (V) which can not only coordinate to a variety of substrates, ${ }^{8}$ but also function as phase transfer catalyst ${ }^{9}$ or $\mathrm{B}-\mathrm{N}$ dehydrogenation catalyst. ${ }^{10}$ Additionally, a few alkylidene-bridged FLPs based on $\mathrm{Ga} / \mathrm{P},{ }^{11} \mathrm{~B} / \mathrm{P}^{12}$ and $\mathrm{Zr} / \mathrm{P}^{13}$ functionalities were reported by Uhl and Erker groups. In spite of the progress in the study of germinal FLPs, the linker is almost exclusively based on carbon. Only very recently, Streubel and coworkers synthesized an anionic oxygen-linked germinal FLP (VI) and investigated its reactivity against $\mathrm{CO}_{2} \cdot{ }^{14}$ Since the nucleophilicity of phosphinite is markedly lower than alkyl- or alkenyl-substituted phosphine, we reasoned that an oxygen-linked germinal FLP containing $-\mathrm{B}\left(\mathrm{C}_{6} \mathrm{~F}_{5}\right)_{2}$ and $-\mathrm{PMes}_{2}$ functionalities (1) would be less susceptible to intramolecular nucleophilic substitution, which could render this FLP stable enough for isolation. Herein, we report the synthesis of oxygen-linked germinal FLP $\mathbf{1}$ and its reactivity against small molecules (Fig. 1).

\section{Results and discussion}

Synthesis of 1 was achieved by treatment of $\mathrm{Mes}_{2} \mathrm{P}(\mathrm{O}) \mathrm{H}$ with $n$ BuLi and subsequently $\left(\mathrm{C}_{6} \mathrm{~F}_{5}\right)_{2} \mathrm{BCl}$ in hexane and it can be isolated as a pale yellow oil with $83 \%$ yield after workup (Scheme 1). Compound 1 was characterized by multinuclear NMR analysis. The ${ }^{31} \mathrm{P}$ NMR spectrum of $\mathbf{1}$ showed a resonance at $\delta 126.4 \mathrm{ppm}$, similar to that observed for phosphinite $\mathrm{Ph}_{2^{-}}$ $\mathrm{P}(\mathrm{OMe})(117 \mathrm{ppm}) \cdot{ }^{15}$ In the ${ }^{11} \mathrm{~B}$ NMR spectrum of $1 \mathrm{a}$ broad 


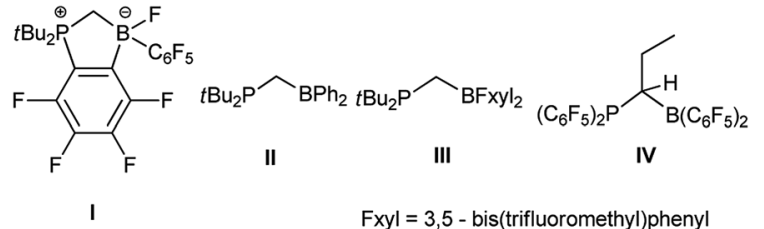

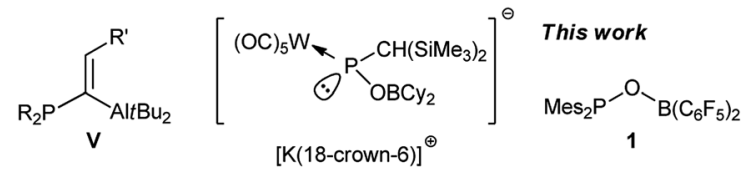

VI

Fig. 1 Germinal intramolecular FLPs.

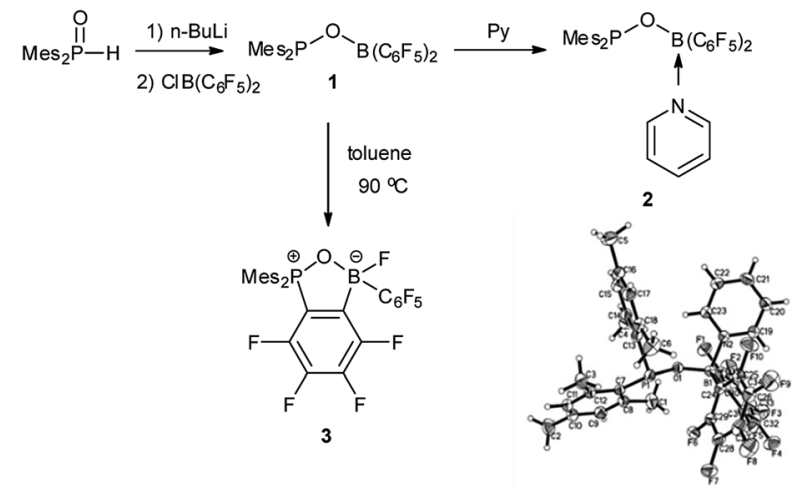

Scheme 1 Synthesis and thermolysis of 1 and molecular structure of 2 (thermal ellipsoids are shown with 30\% probability).

singlet was observed at $\delta 42.4 \mathrm{ppm}$, indicating the existence of a tri-coordinated boron center. This was corroborated by the appearance of signals at $\delta-131.4,-148.8,-161.2 \mathrm{ppm}$ in the ${ }^{19} \mathrm{~F}$ NMR spectrum of $\mathbf{1}$. The identity of $\mathbf{1}$ was further confirmed by derivatization. Addition of equimolar of pyridine to $\mathbf{1}$ in hexane resulted in quantitative formation of adduct 2 , which was characterized by X-ray crystal structure analysis. Compound $\mathbf{1}$ is stable at room temperature both in solution and neat form. Upon heating to $90{ }^{\circ} \mathrm{C}$ in a toluene solution, 1 undergoes intramolecular nucleophilic substitution to afford 3 (Scheme 1), which was isolated in $36 \%$ yield and fully characterized by NMR spectroscopy and X-ray analysis.

To understand the electronic structure of 1, DFT (M06-2X) calculation ${ }^{16}$ was carried out. In the calculated structure, the $\mathrm{P}-\mathrm{O}-\mathrm{B}$ bond angle is $114^{\circ}$ and the distance between the $\mathrm{P}$ and $\mathrm{B}$ atoms is $2.57 \AA$ (Fig. 2a), which is shorter than the observed intramolecular $\mathrm{P} \cdots \mathrm{B}$ distance in $t \mathrm{Bu}_{2} \mathrm{PCH}_{2} \mathrm{~B}(\mathrm{Fxyl})_{2}(2.90 \AA) .{ }^{6}$ Population analysis suggested that the interaction between the $\mathrm{P}$ and $\mathrm{B}$ atoms is very weak and the covalent P-B Wiberg bond order is only 0.02 . The HOMO of $\mathbf{1}$ mainly corresponds to the lone pair on phosphorus, with some contribution from $\pi$ orbital of the mesityl substitutent (Fig. 2b). The LUMO is largely distributed over the boron center as well as one $\mathrm{C}_{6} \mathrm{~F}_{5}$ ring and the oxygen linker, indicative of $\pi$ electron donation from the oxygen atom to the empty p orbital of the boron center (Fig. 2c).

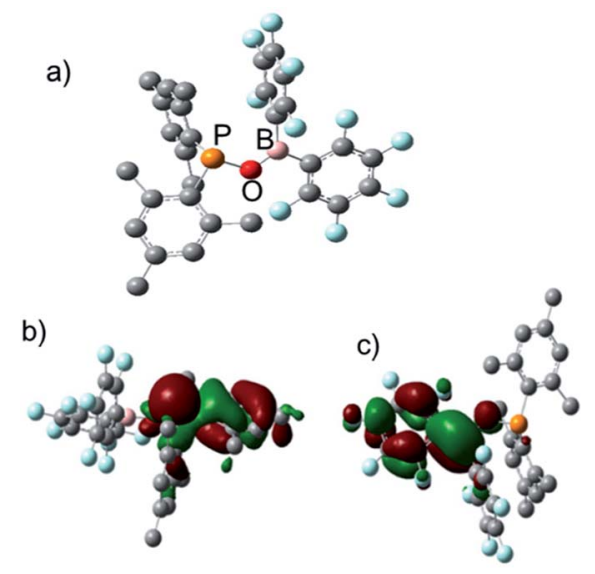

Fig. 2 (a) calculated structure of 1; (b) HOMO of 1; (c) LUMO of 1.

While 1 does not react with $\mathrm{H}_{2}$ under 6 bar at room temperature, it reacts with $\mathrm{H}_{2}$ under harsher conditions $\left(60 \mathrm{bar}, 50^{\circ} \mathrm{C}\right)$ to afford compound 4 with $58 \%$ yield after workup (Scheme 2). Compound 4 can be also independently synthesized from $\mathrm{Mes}_{2} \mathrm{P}(\mathrm{O}) \mathrm{H}$ and $\mathrm{HB}\left(\mathrm{C}_{6} \mathrm{~F}_{5}\right)_{2}$. Existence of a P-H moiety was confirmed by the appearance of a doublet at $\delta 31.5 \mathrm{ppm}\left({ }^{1} J_{\mathrm{P}-\mathrm{H}}=\right.$ $514 \mathrm{~Hz}$ ) in the ${ }^{31} \mathrm{P}$ NMR spectrum, with a corresponding doublet at $\delta 8.02 \mathrm{ppm}\left({ }^{1} \mathrm{~J}_{\mathrm{P}-\mathrm{H}}=514 \mathrm{~Hz}\right)$ observed in the ${ }^{1} \mathrm{H}$ NMR spectrum. However, both the ${ }^{11} \mathrm{~B}$ and ${ }^{11} \mathrm{~B}\left\{{ }^{1} \mathrm{H}\right\}$ NMR spectra displayed a broad signal at $-4.79 \mathrm{ppm}$, excluding study of the coupling constant with the adjacent $\mathrm{H}$ atom. The ${ }^{1} \mathrm{H}$ NMR spectrum displayed a broad singlet at $\delta 4.59 \mathrm{ppm}$ with integration of 1 , which was assigned to the $\mathrm{B} H$ moiety. The identity of $\mathbf{4}$ was further validated by X-ray crystal structure analysis (Fig. 3).

Treatment of 1 with $\mathrm{CO}_{2}(1.8 \mathrm{bar})$ at room temperature in hexane resulted in formation of a $\mathrm{CO}_{2}$ adduct 5 , in an analogous way with other germinal FLPs (Scheme 3)..$^{5 a, 6,8 a, 11,13 b, 14} \mathbf{5}$ was characterized by both NMR spectroscopy and X-ray analysis (Fig. 4). 5 is stable infinitely as solid. But unlike germinal B/P FLPs II $^{5 a}$ and $\mathbf{I I I}{ }^{6}$ the solution of 5 in $\mathrm{C}_{6} \mathrm{D}_{6}$ slowly released $\mathrm{CO}_{2}$ under $\mathrm{N}_{2}$ atmosphere at room temperature. In a sealed

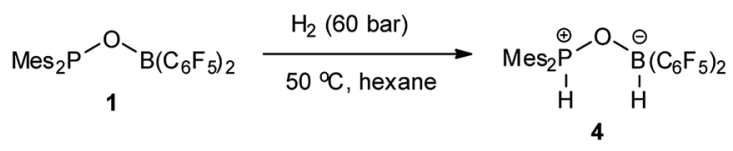

Scheme 2 Reaction of 1 with $\mathrm{H}_{2}$.

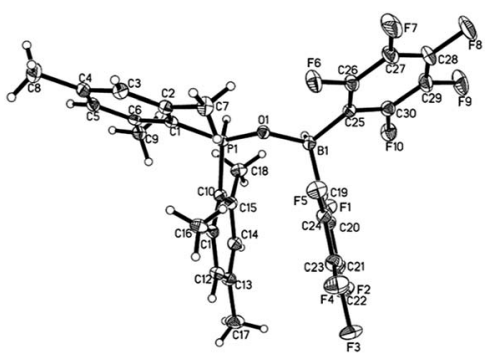

Fig. 3 Molecular structure of 4 (thermal ellipsoids are shown with $30 \%$ probability). 


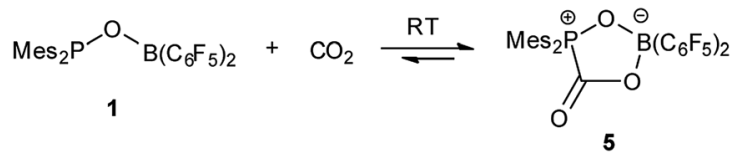

Scheme 3 Reaction of 1 with $\mathrm{CO}_{2}$.

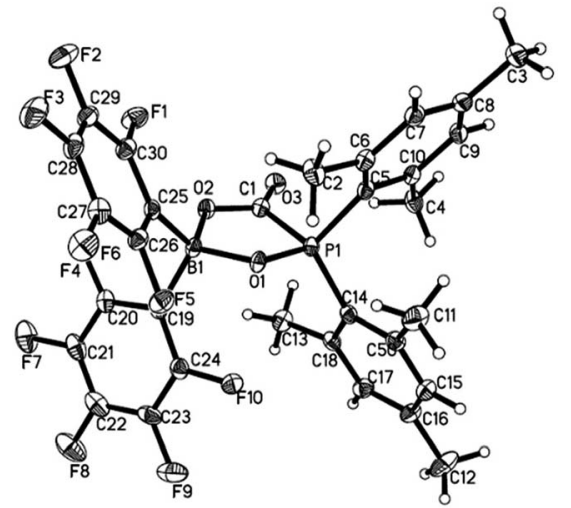

Fig. 4 Molecular structure of 5 (thermal ellipsoids are shown with 30\% probability).

NMR tube at room temperature, equilibrium was reached after 24 hours and about $25 \%$ of $\mathbf{5}$ was converted to $\mathbf{1}$.

Reaction of 1 with phenylacetylene in hexane led to addition of both phosphoryl and boryl moieties to the $\mathrm{C} \equiv \mathrm{C}$ bond to give 6 in $73 \%$ yield (Scheme 4). This is in line with the discovery by Stephan ${ }^{17}$ and Erker ${ }^{7}$ that FLPs with phosphine moiety of low basicity tend to afford addition products. In the solid structure of 6 (Fig. 5), the distances of $\mathrm{B}-\mathrm{O}$ and $\mathrm{P}-\mathrm{O}$ are similar to those observed in $t \mathrm{Bu}_{2} \mathrm{P}(\mu-\mathrm{O})\left(\mu-\mathrm{C}_{6} \mathrm{H}_{4}\right) \mathrm{B}\left(\mathrm{C}_{6} \mathrm{~F}_{5}\right)_{2}(\mathrm{P}-\mathrm{O} 1.546, \mathrm{~B}-\mathrm{O} 1.550 \AA)$ reported by Wagner. ${ }^{6}$

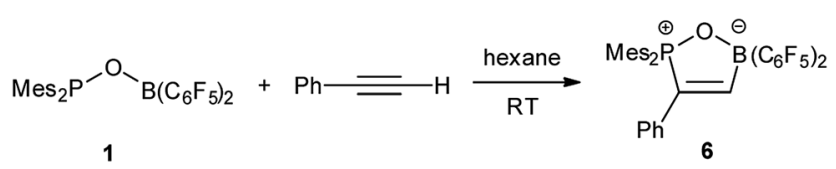

Scheme 4 Reaction of 1 with phenylacetylene.

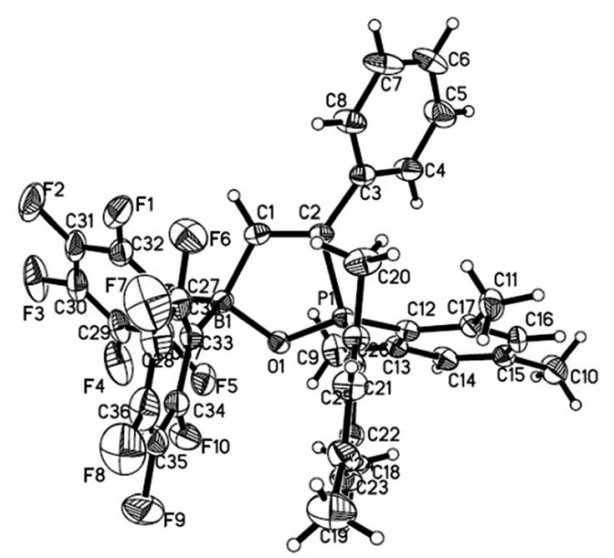

Fig. 5 Molecular structure of 6 (thermal ellipsoids are shown with $30 \%$ probability).

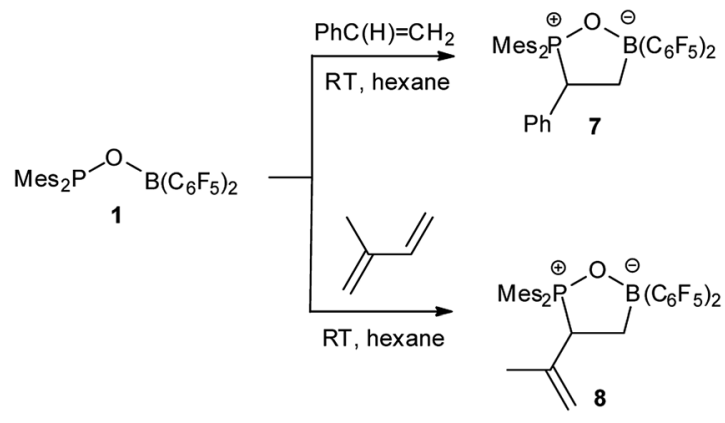

Scheme 5 Reaction of 1 with styrene and isoprene.

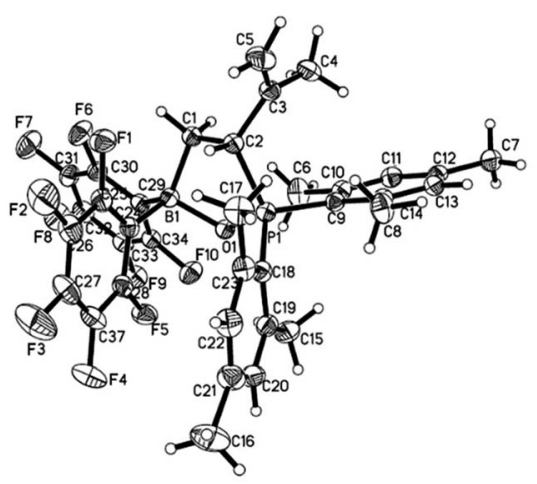

Fig. 6 Molecular structure of 8 (thermal ellipsoids are shown with 30\% probability).

Compound 1 reacted with equimolar of styrene in hexane at room temperature to yield a white precipitate, which was identified as 1,2-boryl/phosphoryl addition product 7 via NMR spectroscopy and X-ray analysis. ${ }^{18}$ When 1 was treated with isoprene in hexane, 3,4-phosphoryl/boryl addition product $\mathbf{8}$ was formed as the only regioisomer, which can be isolated as a white solid in $79 \%$ yield (Scheme 5). 8 was characterized by both NMR spectroscopy and X-ray analysis (Fig. 6). This observed regioselectivity is in contrast to previous reports by Stephan $^{\mathbf{1 9}}$ and Lerner ${ }^{\mathbf{2 0}}$ that activation of dienes with intermolecular FLP $t \mathrm{Bu}_{3} \mathrm{P} / \mathrm{B}\left(\mathrm{C}_{6} \mathrm{~F}_{5}\right)_{3}$ or intramolecular FLP di- $t$-butylphosphaboradibenzofulvene affords predominately 1,4-addition products. It is likely that in the case of intramolecular FLP 1, the favored formation of a five-member ring dictates the observed 3,4-selectivity.

\section{Conclusions}

By employing an oxygen atom as the linker, a germinal FLP containing both electrophilic $\mathrm{B}\left(\mathrm{C}_{6} \mathrm{~F}_{5}\right)_{2}$ and nucleophilic $\mathrm{PMes}_{2}$ moieties was synthesized for the first time. This intramolecular FLP readily reacts with a series of small molecules, such as $\mathrm{H}_{2}$, $\mathrm{CO}_{2}$, phenylacetylene, styrene and isoprene. Among them, regioselective 3,4-addition to a diene is unprecedented. Current research is focusing on deoxygenation of addition adducts, which might provide a novel way for the synthesis of intramolecular FLPs. 


\section{Experimental section}

\section{General experimental methods}

Solvents were dried by reflux under $\mathrm{N}_{2}$ over sodium or $\mathrm{CaH}_{2}$ and freshly distilled prior to use. Air-sensitive compounds were handled under a $\mathrm{N}_{2}$ atmosphere using standard Schlenk and glovebox techniques. NMR spectra were recorded on Bruker SPECT NMR (400 MHz for ${ }^{1} \mathrm{H}, 376 \mathrm{MHz}$ for ${ }^{19} \mathrm{~F}, 100 \mathrm{MHz}$ for ${ }^{13} \mathrm{C}$ ) and Bruker DMX500 NMR (500 MHz for ${ }^{1} \mathrm{H}, 160 \mathrm{MHz}$ for ${ }^{11} \mathrm{~B}$ ) spectrometers. Most assignments were based on a series of $2 \mathrm{D}$ NMR experiments. HRMS analyses were performed at Bruker micrOTOF II. Crystallographic data for the structure reported in this paper have been deposited with the Cambridge Crystallographic Data Center: CCDC 1843771-1843777 (compound 2-8) contain the supplementary crystallographic data for this paper. These data can be obtained free of charge from The Cambridge Crystallographic Data Centre at www.ccdc.cam.ac.uk/ data_request/cif. $\mathrm{Mes}_{2} \mathrm{P}(\mathrm{O}) \mathrm{H}\left(\mathrm{Mes}=\right.$ 2,4,6-trimethylphenyl) ${ }^{21}$ and $\operatorname{ClB}\left(\mathrm{C}_{6} \mathrm{~F}_{5}\right)_{2}$ (ref. 22) were prepared as reported.

\section{Synthesis of compound 1}

A solution of $n$-BuLi $(2.5 \mathrm{M}, 0.80 \mathrm{~mL}, 2.0 \mathrm{mmol})$ was added to a suspension of $\mathrm{Mes}_{2} \mathrm{P}(\mathrm{O}) \mathrm{H}(0.57 \mathrm{~g}, 2.0 \mathrm{mmol})$ in hexane $(8 \mathrm{~mL})$ at $0{ }^{\circ} \mathrm{C}$, affording a pale yellow solution. After stirring for $4 \mathrm{~h}$ at room temperature, a solution of $\mathrm{ClB}\left(\mathrm{C}_{6} \mathrm{~F}_{5}\right)_{2}(0.76 \mathrm{~g}, 2.0 \mathrm{mmol})$ in hexane $(6 \mathrm{~mL})$ was added at $0{ }^{\circ} \mathrm{C}$. The reaction mixture was warmed up to room temperature and stirred for $12 \mathrm{~h}$. The resulting white slurry was filtered and the filtrate was dried under vacuum affording 1 as a pale yellow oil $(1.05 \mathrm{~g}, 81 \%) .{ }^{1} \mathrm{H}$ NMR (400 MHz, $\left.\mathrm{C}_{6} \mathrm{D}_{6}\right): \delta[\mathrm{ppm}]=6.62\left(\mathrm{~d}, 4 \mathrm{H},{ }^{4} J_{\mathrm{P}-\mathrm{H}}=2 \mathrm{~Hz}\right.$, metaMes), 2.28 (s, $12 \mathrm{H}$, ortho- $\left.\mathrm{CH}_{3}\right), 2.02\left(\mathrm{~s}, 6 \mathrm{H}\right.$, para- $\left.\mathrm{CH}_{3}\right) .{ }^{13} \mathrm{C} \mathrm{NMR}$ $\left(101 \mathrm{MHz}, \mathrm{C}_{6} \mathrm{D}_{6}\right): \delta[\mathrm{ppm}]=147.9\left(\mathrm{dm},{ }^{1} J_{\mathrm{C}-\mathrm{F}}=248 \mathrm{~Hz}\right.$, ortho$\left.C_{6} \mathrm{~F}_{5}\right), 143.2\left(\mathrm{dm},{ }^{1} J_{\mathrm{C}-\mathrm{F}}=257 \mathrm{~Hz}\right.$, meta $\left.-C_{6} \mathrm{~F}_{5}\right), 137.6\left(\mathrm{dm},{ }^{1} J_{\mathrm{C}-\mathrm{F}}=\right.$ $253 \mathrm{~Hz}$, para $-C_{6} \mathrm{~F}_{5}$ ), 141.3 (d, ${ }^{2} J_{\mathrm{P}-\mathrm{C}}=17 \mathrm{~Hz}$, ortho-Mes), 140.2 (para-Mes), 133.3 (d, ${ }^{1} J_{\mathrm{P}-\mathrm{C}}=28 \mathrm{~Hz}$, ipso-Mes), $130.6\left(\mathrm{~d},{ }^{3} J_{\mathrm{P}-\mathrm{C}}=\right.$ $3 \mathrm{~Hz}$, meta-Mes), $22.2\left(\mathrm{~d},{ }^{3} \mathrm{~J}_{\mathrm{P}-\mathrm{C}}=4 \mathrm{~Hz}\right.$, ortho- $\mathrm{CH}_{3}$ ), 20.9 (para$\left.\mathrm{CH}_{3}\right) .{ }^{19} \mathrm{~F}$ NMR $\left(376 \mathrm{MHz}, \mathrm{C}_{6} \mathrm{D}_{6}\right): \delta[\mathrm{ppm}]=-131.4(\mathrm{~m}$, ortho$\left.\mathrm{C}_{6} F_{5}\right),-148.8\left(\mathrm{t}\right.$, para $\left.-\mathrm{C}_{6} F_{5}\right),-161.2\left(\mathrm{~m}\right.$, meta $\left.-\mathrm{C}_{6} F_{5}\right) .{ }^{31} \mathrm{P} \mathrm{NMR}$ $\left(162 \mathrm{MHz}, \mathrm{C}_{6} \mathrm{D}_{6}\right): \delta[\mathrm{ppm}]=126.4 .{ }^{11} \mathrm{~B} \mathrm{NMR}\left(160 \mathrm{MHz}, \mathrm{C}_{6} \mathrm{D}_{6}\right)$ : $\delta[\mathrm{ppm}]=42.4$. HRMS $(\mathrm{m} / \mathrm{z})$ : calcd for $\mathrm{C}_{30} \mathrm{H}_{23} \mathrm{OPBF}_{10}[\mathrm{M}+\mathrm{H}]^{+}$: 631.1420, found: 631.1434 .

\section{Synthesis of compound 2}

A solution of pyridine ( $25 \mathrm{mg}, 0.32 \mathrm{mmol})$ in hexane $(1 \mathrm{~mL})$ was added to a solution of 1 (130 $\mathrm{mg}, 0.206 \mathrm{mmol})$ in hexane $(2 \mathrm{~mL})$, affording a white precipitate. The reaction mixture was stirred for $30 \mathrm{~min}$ and stored at $-30{ }^{\circ} \mathrm{C}$ for $10 \mathrm{~h}$. Then the supernatant was removed by filtration and the residue was washed with hexane $(1 \mathrm{~mL})$ and dried under vacuum, affording 2 as a white powder (118 mg, 81\%). Crystals suitable for X-ray diffraction were grown by slow diffusion of hexane into a solution of 2 in $\mathrm{CH}_{2} \mathrm{Cl}_{2} \cdot{ }^{1} \mathrm{H}$ NMR (400 MHz, $\left.\mathrm{C}_{6} \mathrm{D}_{6}\right): \delta[\mathrm{ppm}]=8.12(\mathrm{br} \mathrm{s}, 2 \mathrm{H}$, ortho-Py), 6.66 (s, 4H, meta-Mes), 6.54 (br s, 1H, para-Py), 6.21 (br s, 2H, meta-Py), 2.47 (s, 12H, ortho- $\left.\mathrm{CH}_{3}\right), 2.10$ (s, 6H, para$\left.\mathrm{CH}_{3}\right) \cdot{ }^{13} \mathrm{C} \mathrm{NMR}\left(101 \mathrm{MHz}, \mathrm{C}_{6} \mathrm{D}_{6}\right): \delta[\mathrm{ppm}]=148.6\left(\mathrm{dm},{ }^{1} J_{\mathrm{C}-\mathrm{F}}=\right.$ $240 \mathrm{~Hz}$, ortho $\left.-C_{6} \mathrm{~F}_{5}\right), 140.8\left(\mathrm{dm},{ }^{1} J_{\mathrm{C}-\mathrm{F}}=251 \mathrm{~Hz}\right.$, meta $\left.-C_{6} \mathrm{~F}_{5}\right), 137.8$ (dm, ${ }^{1} J_{\mathrm{C}-\mathrm{F}}=251 \mathrm{~Hz}$, para- $C_{6} \mathrm{~F}_{5}$ ), 144.3 (ortho-Py), 141.0 (para-Py), 125.0 (meta-Py), 141.5 (d, ${ }^{2} J_{\mathrm{P}-\mathrm{C}}=17 \mathrm{~Hz}$, ortho-Mes), 138.3 (paraMes), 136.9 (d, ${ }^{1} J_{\mathrm{P}-\mathrm{C}}=32 \mathrm{~Hz}$, ipso-Mes), 130.5 (d, ${ }^{3} J_{\mathrm{P}-\mathrm{C}}=2 \mathrm{~Hz}$, meta-Mes), 21.5 (d, ${ }^{3} J_{\mathrm{P}-\mathrm{C}}=16 \mathrm{~Hz}$, ortho- $\left.\mathrm{CH}_{3}\right), 20.9$ (para- $\left.\mathrm{CH}_{3}\right)$. ${ }^{19} \mathrm{~F}$ NMR $\left(376 \mathrm{MHz}, \mathrm{C}_{6} \mathrm{D}_{6}\right): \delta[\mathrm{ppm}]=-131.6\left(\mathrm{~m}\right.$, ortho $\left.-\mathrm{C}_{6} \mathrm{~F}_{5}\right)$, -155.8 (t, para $\left.-\mathrm{C}_{6} \mathrm{~F}_{5}\right),-163.3\left(\mathrm{~m}\right.$, meta $\left.-\mathrm{C}_{6} \mathrm{~F}_{5}\right) .{ }^{31} \mathrm{P}$ NMR (162 $\left.\mathrm{MHz}, \mathrm{C}_{6} \mathrm{D}_{6}\right): \delta[\mathrm{ppm}]=102.9 .{ }^{11} \mathrm{~B} \mathrm{NMR}\left(160 \mathrm{MHz}, \mathrm{C}_{6} \mathrm{D}_{6}\right): \delta[\mathrm{ppm}]$ $=4.37$.

\section{Synthesis of compound 3}

A solution of $1(132 \mathrm{mg}, 0.206 \mathrm{mmol})$ in toluene $(1 \mathrm{~mL})$ was heated to $90{ }^{\circ} \mathrm{C}$ for $15 \mathrm{~h}$. Then, all volatiles were removed under vacuum. The resulting oily residue was extracted with hexane (2 $\mathrm{mL}$ ). Afterwards hexane was removed under vacuum affording an oily solid. Another portion of hexane $(1 \mathrm{~mL})$ was introduced and an insoluble oil was formed which became crystals after standing at room temperature for $30 \mathrm{~min}$. The supernatant was removed and the crystals were dried under vacuum affording 3 as a white crystalline solid (47 mg, 36\%). ${ }^{1} \mathrm{H}$ NMR (400 MHz, $\left.\mathrm{C}_{6} \mathrm{D}_{6}\right): \delta[\mathrm{ppm}]=6.43,6.27$ (each d, each $2 \mathrm{H},{ }^{4} J_{\mathrm{P}-\mathrm{H}}=5 \mathrm{~Hz}$, metaMes), 2.19, 2.05 (each s, each $6 \mathrm{H}$, ortho- $\mathrm{CH}_{3}$ ), 1.84, 1.71 (each s, each $3 \mathrm{H}$, para- $\left.\mathrm{CH}_{3}\right) .{ }^{13} \mathrm{C} \mathrm{NMR}\left(101 \mathrm{MHz}, \mathrm{C}_{6} \mathrm{D}_{6}\right): \delta[\mathrm{ppm}]=145.2$, $144.6\left(\mathrm{~d},{ }^{4} \mathrm{~J}_{\mathrm{P}-\mathrm{C}}=3 \mathrm{~Hz}\right.$, para-Mes), $143.5,141.8$ (d, ${ }^{2} J_{\mathrm{P}-\mathrm{C}}=12 \mathrm{~Hz}$, ortho-Mes), 132.4, 131.5 (d, ${ }^{3} J_{\mathrm{P}-\mathrm{C}}=13 \mathrm{~Hz}$, meta-Mes), 23.1, 21.8 $\left(\mathrm{d},{ }^{3} \mathrm{~J}_{\mathrm{P}-\mathrm{C}}=5 \mathrm{~Hz}\right.$, ortho- $\left.\mathrm{CH}_{3}\right), 20.9,20.7\left(\mathrm{~d},{ }^{5} J_{\mathrm{P}-\mathrm{C}}=1 \mathrm{~Hz}, \mathrm{p}-\mathrm{CH}_{3}\right)$. ${ }^{19} \mathrm{~F}$ NMR $\left(376 \mathrm{MHz}, \mathrm{C}_{6} \mathrm{D}_{6}\right): \delta[\mathrm{ppm}]=-129.5\left(\mathrm{~m}, 2 \mathrm{~F}, \mathrm{C}_{6} F_{4}\right)$, $-134.5\left(\mathrm{~m}, 2 \mathrm{~F}\right.$, ortho- $\left.\mathrm{C}_{6} F_{5}\right),-144.1\left(\mathrm{~m}, 1 \mathrm{~F}, \mathrm{C}_{6} F_{4}\right),-149.5$ (br s, $1 \mathrm{~F}, \mathrm{BF}),-153.6\left(\mathrm{~m}, 1 \mathrm{~F}, \mathrm{C}_{6} F_{4}\right),-158.0\left(\mathrm{t}, 1 \mathrm{~F}\right.$, para- $\left.\mathrm{C}_{6} F_{5}\right),-164.6$ $\left(\mathrm{m}, 2 \mathrm{~F}\right.$, meta- $\left.\mathrm{C}_{6} F_{5}\right) .{ }^{31} \mathrm{P} \mathrm{NMR}\left(162 \mathrm{MHz}, \mathrm{C}_{6} \mathrm{D}_{6}\right): \delta[\mathrm{ppm}]=58.3$. ${ }^{11} \mathrm{~B}$ NMR $\left(160 \mathrm{MHz}, \mathrm{C}_{6} \mathrm{D}_{6}\right): \delta[\mathrm{ppm}]=8.72$. HRMS $(\mathrm{m} / \mathrm{z})$ : calcd for $\mathrm{C}_{30} \mathrm{H}_{22} \mathrm{OPBF}_{10} \mathrm{Na}[\mathrm{M}+\mathrm{Na}]^{+}:$653.1239, found: 653.1258 .

\section{Synthesis of compound 4}

$\mathrm{H}_{2}$ (60 bar) was introduced to a solution of 1 (324 mg, 0.515 $\mathrm{mmol})$ in hexane $(2.5 \mathrm{~mL})$ in an autoclave. The reaction mixture was stirred for $40 \mathrm{~h}$ at $50{ }^{\circ} \mathrm{C}$, affording a white precipitate. The supernatant was removed by filtration and the solid was dried under vacuum affording 4 as a white powder (188 $\mathrm{mg}, 58 \%$ ). Crystals suitable for X-ray diffraction were grown by slow diffusion of hexane into a solution of 4 in toluene. ${ }^{1} \mathrm{H}$ NMR (400 $\left.\mathrm{MHz}, \mathrm{C}_{6} \mathrm{D}_{6}\right): \delta[\mathrm{ppm}]=8.02\left(\mathrm{~d}, 1 \mathrm{H},{ }^{1} J_{\mathrm{P}-\mathrm{H}}=514 \mathrm{~Hz}, \mathrm{PH}\right), 6.34(\mathrm{~d}$, $4 \mathrm{H},{ }^{4} \mathrm{~J}_{\mathrm{P}-\mathrm{H}}=5 \mathrm{~Hz}$, meta-Mes), 4.59 (br s, $\left.1 \mathrm{H}, \mathrm{BH}\right), 2.07(\mathrm{~s}, 12 \mathrm{H}$, ortho- $\left.\mathrm{CH}_{3}\right), 1.86\left(\mathrm{~s}, 6 \mathrm{H}\right.$, para- $\left.\mathrm{CH}_{3}\right) .{ }^{13} \mathrm{C} \mathrm{NMR}\left(101 \mathrm{MHz}, \mathrm{C}_{6} \mathrm{D}_{6}\right)$ : $\delta[\mathrm{ppm}]=148.4\left(\mathrm{dm},{ }^{1} J_{\mathrm{C}-\mathrm{F}}=240 \mathrm{~Hz}\right.$, ortho- $\left.C_{6} \mathrm{~F}_{5}\right), 139.9\left(\mathrm{dm},{ }^{1} J_{\mathrm{C}-}\right.$ $\mathrm{F}=247 \mathrm{~Hz}$, meta $\left.-C_{6} \mathrm{~F}_{5}\right), 137.4\left(\mathrm{dm},{ }^{1} J_{\mathrm{C}-\mathrm{F}}=256 \mathrm{~Hz}\right.$, para- $\left.C_{6} \mathrm{~F}_{5}\right)$, $145.0\left(\mathrm{~d},{ }^{4} \mathrm{~J}_{\mathrm{P}-\mathrm{C}}=3 \mathrm{~Hz}\right.$, para-Mes), $143.1\left(\mathrm{~d},{ }^{2} J_{\mathrm{P}-\mathrm{C}}=11 \mathrm{~Hz}\right.$, orthoMes), 131.2 (d, ${ }^{3} J_{\mathrm{P}-\mathrm{C}}=12 \mathrm{~Hz}$, meta-Mes), 117.8 (d, ${ }^{1} J_{\mathrm{P}-\mathrm{C}}=$ $107 \mathrm{~Hz}$, ipso-Mes), 21.0 (d, ${ }^{3} J_{\mathrm{P}-\mathrm{C}}=6 \mathrm{~Hz}$, ortho- $\left.\mathrm{CH}_{3}\right), 20.9\left(\mathrm{~d},{ }^{5} J_{\mathrm{P}-\mathrm{C}}\right.$ $=2 \mathrm{~Hz}$, para- $\left.\mathrm{CH}_{3}\right) .{ }^{19} \mathrm{~F} \mathrm{NMR}\left(376 \mathrm{MHz}, \mathrm{C}_{6} \mathrm{D}_{6}\right): \delta[\mathrm{ppm}]=-133.9$ $\left(\mathrm{m}\right.$, ortho- $\left.\mathrm{C}_{6} F_{5}\right),-159.8\left(\mathrm{t}\right.$, para $\left.-\mathrm{C}_{6} F_{5}\right),-165.1\left(\mathrm{~m}\right.$, meta $\left.-\mathrm{C}_{6} F_{5}\right) .{ }^{31} \mathrm{P}$ $\operatorname{NMR}\left(162 \mathrm{MHz}, \mathrm{C}_{6} \mathrm{D}_{6}\right): \delta[\mathrm{ppm}]=31.5\left(\mathrm{~d},{ }^{1} J_{\mathrm{P}-\mathrm{H}}=514 \mathrm{~Hz}\right) \cdot{ }^{11} \mathrm{~B}$ NMR $\left(160 \mathrm{MHz}, \mathrm{C}_{6} \mathrm{D}_{6}\right): \delta[\mathrm{ppm}]=-4.79$.

\section{Synthesis of compound 5}

$\mathrm{CO}_{2}$ (1.8 bar) was introduced to a degassed solution of 1 (551 $\mathrm{mg}, 0.875 \mathrm{mmol})$ in hexane $(2.5 \mathrm{~mL})$ at room temperature 
and the reaction mixture was stirred for $24 \mathrm{~h}$, affording a white precipitate. The supernatant was removed by filtration and the residue was dried under vacuum affording $\mathbf{5}$ as a white powder (430 mg, 73\%). Crystals suitable for X-ray diffraction were grown from toluene at $-30{ }^{\circ} \mathrm{C} .{ }^{1} \mathrm{H}$ NMR $\left(400 \mathrm{MHz}, \mathrm{C}_{6} \mathrm{D}_{6}\right): \delta[\mathrm{ppm}]=$ $6.30\left(\mathrm{~s}, 4 \mathrm{H}\right.$, meta-Mes), $2.19\left(\mathrm{~s}, 12 \mathrm{H}\right.$, ortho- $\left.\mathrm{CH}_{3}\right), 1.79(\mathrm{~s}, 6 \mathrm{H}$, para- $\left.\mathrm{CH}_{3}\right) .{ }^{13} \mathrm{C}$ NMR $\left(101 \mathrm{MHz}, \mathrm{C}_{6} \mathrm{D}_{6}\right): \delta[\mathrm{ppm}]=165.9\left(\mathrm{~d},{ }^{1} J_{\mathrm{P}-\mathrm{C}}\right.$ $=108 \mathrm{~Hz}, C=\mathrm{O}), 148.4\left(\mathrm{dm},{ }^{1} J_{\mathrm{C}-\mathrm{F}}=243 \mathrm{~Hz}\right.$, ortho- $\left.C_{6} \mathrm{~F}_{5}\right), 141.0$ $\left(\mathrm{dm},{ }^{1} J_{\mathrm{C}-\mathrm{F}}=253 \mathrm{~Hz}\right.$, meta- $\left.C_{6} \mathrm{~F}_{5}\right), 137.6\left(\mathrm{dm},{ }^{1} J_{\mathrm{C}-\mathrm{F}}=251 \mathrm{~Hz}\right.$, para$C_{6} \mathrm{~F}_{5}$ ), 145.7 (para-Mes), 143.3 (d, ${ }^{2} J_{\mathrm{P}-\mathrm{C}}=12 \mathrm{~Hz}$, ortho-Mes), 131.7 (d, ${ }^{3} J_{\mathrm{P}-\mathrm{C}}=13 \mathrm{~Hz}$, meta-Mes), 118.0 (d, ${ }^{1} J_{\mathrm{P}-\mathrm{C}}=92 \mathrm{~Hz}$, ipsoMes), $21.8\left(\mathrm{~d},{ }^{3} \mathrm{~J}_{\mathrm{P}-\mathrm{C}}=6 \mathrm{~Hz}\right.$, ortho- $\left.\mathrm{CH}_{3}\right), 20.9\left(\right.$ para $\left.-\mathrm{CH}_{3}\right) .{ }^{19} \mathrm{~F} \mathrm{NMR}$ $\left(376 \mathrm{MHz}, \mathrm{C}_{6} \mathrm{D}_{6}\right): \delta[\mathrm{ppm}]=-133.9\left(\right.$ ortho- $\left.\mathrm{C}_{6} F_{5}\right),-155.9$ (para$\left.\mathrm{C}_{6} F_{5}\right),-163.5\left(\right.$ meta- $\left.\mathrm{C}_{6} F_{5}\right) .{ }^{31} \mathrm{P} \mathrm{NMR}\left(162 \mathrm{MHz}, \mathrm{C}_{6} \mathrm{D}_{6}\right): \delta[\mathrm{ppm}]=$ 35.0. ${ }^{11} \mathrm{~B}$ NMR $\left(160 \mathrm{MHz}, \mathrm{C}_{6} \mathrm{D}_{6}\right): \delta[\mathrm{ppm}]=7.94$. HRMS $(\mathrm{m} / \mathrm{z})$ : calcd for $\mathrm{C}_{31} \mathrm{H}_{22} \mathrm{O}_{3} \mathrm{PBF}_{10} \mathrm{Na}[\mathrm{M}+\mathrm{Na}]^{+}$: 697.1138, found: 697.1123

\section{Synthesis of compound 6}

$\mathrm{PhC} \equiv \mathrm{CH}$ (120 mg, $1.18 \mathrm{mmol}$ ) was added to a solution of $\mathbf{1}$ $(567 \mathrm{mg}, 0.900 \mathrm{mmol})$ in hexane $(3 \mathrm{~mL})$ at room temperature, affording a white precipitate. After the reaction mixture was stirred for $17 \mathrm{~h}$, the supernatant was removed by filtration and the solid was dried under vacuum affording 6 as a white powder (480 mg, 73\%). Crystals suitable for X-ray diffraction were grown by slow diffusion of hexane into a solution of 6 in toluene. ${ }^{1} \mathrm{H}$ NMR (400 MHz, $\left.\mathrm{C}_{6} \mathrm{D}_{6}\right): \delta[\mathrm{ppm}]=9.01\left(\mathrm{~d}, 1 \mathrm{H},{ }^{3} J_{\mathrm{P}-\mathrm{H}}=46 \mathrm{~Hz}, \mathrm{C}=\right.$ $\mathrm{CH}), 7.17(\mathrm{~m}, 2 \mathrm{H}, \mathrm{Ph} H), 6.94(\mathrm{~m}, 3 \mathrm{H}, \mathrm{Ph} H), 6.40\left(\mathrm{~d}, 4 \mathrm{H},{ }^{4} J_{\mathrm{P}-\mathrm{H}}=\right.$ $4 \mathrm{~Hz}$, meta-Mes), 2.10 (s, $12 \mathrm{H}$, ortho- $\left.\mathrm{CH}_{3}\right), 1.85$ (s, 6H, para- $\mathrm{CH}_{3}$ ). ${ }^{13} \mathrm{C}$ NMR (101 MHz, $\left.\mathrm{C}_{6} \mathrm{D}_{6}\right): \delta[\mathrm{ppm}]=176.7$ (br s, BCH), 147.8 $\left(\mathrm{dm},{ }^{1} J_{\mathrm{C}-\mathrm{F}}=241 \mathrm{~Hz}\right.$, ortho- $\left.C_{6} \mathrm{~F}_{5}\right), 139.9\left(\mathrm{dm},{ }^{1} J_{\mathrm{C}-\mathrm{F}}=250 \mathrm{~Hz}\right.$, meta- $\left.C_{6} \mathrm{~F}_{5}\right), 137.1\left(\mathrm{dm},{ }^{1} J_{\mathrm{C}-\mathrm{F}}=254 \mathrm{~Hz}\right.$, para $\left.-C_{6} \mathrm{~F}_{5}\right), 144.0\left(\mathrm{~d},{ }^{4} J_{\mathrm{P}-\mathrm{C}}\right.$ $=3 \mathrm{~Hz}$, para-Mes), 142.7 (d, ${ }^{2} J_{\mathrm{P}-\mathrm{C}}=11 \mathrm{~Hz}$, ortho-Mes), 136.7 (d, ${ }^{2} J_{\mathrm{P}-\mathrm{C}}=21 \mathrm{~Hz}$, ipso-Ph), $135.2\left(\mathrm{~d},{ }^{1} J_{\mathrm{P}-\mathrm{C}}=81 \mathrm{~Hz}, \mathrm{PC}=\mathrm{C}\right), 131.5(\mathrm{~d}$, ${ }^{3} J_{\mathrm{P}-\mathrm{C}}=12 \mathrm{~Hz}$, meta-Mes), $129.2(\mathrm{Ph}), 127.5\left(\mathrm{~d},{ }^{3} J_{\mathrm{P}-\mathrm{C}}=5 \mathrm{~Hz}\right.$, ortho-Ph), 124.9 (d, ${ }^{1} J_{\mathrm{P}-\mathrm{C}}=97 \mathrm{~Hz}$, ipso-Mes), 23.0 (d, ${ }^{3} \mathrm{~J}_{\mathrm{P}-\mathrm{C}}=$ $5 \mathrm{~Hz}$, ortho- $\left.\mathrm{CH}_{3}\right), 20.8\left(\mathrm{~d},{ }^{5} \mathrm{~J}_{\mathrm{P}-\mathrm{C}}=1 \mathrm{~Hz}\right.$, para- $\left.\mathrm{CH}_{3}\right) .{ }^{19} \mathrm{~F}$ NMR $(376$ $\left.\mathrm{MHz}, \mathrm{C}_{6} \mathrm{D}_{6}\right): \delta[\mathrm{ppm}]=-132.0\left(\mathrm{~m}\right.$, ortho- $\left.\mathrm{C}_{6} F_{5}\right),-159.2(\mathrm{t}$, para$\left.\mathrm{C}_{6} F_{5}\right),-164.5\left(\mathrm{~m}\right.$, meta- $\left.\mathrm{C}_{6} F_{5}\right) .{ }^{31} \mathrm{P} \mathrm{NMR}\left(162 \mathrm{MHz}, \mathrm{C}_{6} \mathrm{D}_{6}\right): \delta[\mathrm{ppm}]$ $=68.4\left(\mathrm{~d},{ }^{3} J_{\mathrm{P}-\mathrm{H}}=46 \mathrm{~Hz}\right) \cdot{ }^{11} \mathrm{~B} \mathrm{NMR}\left(160 \mathrm{MHz}, \mathrm{C}_{6} \mathrm{D}_{6}\right): \delta[\mathrm{ppm}]=$ 2.65. HRMS $(\mathrm{m} / \mathrm{z})$ : calcd for $\mathrm{C}_{38} \mathrm{H}_{28} \mathrm{OPBF}_{10} \mathrm{Na}[\mathrm{M}+\mathrm{Na}]^{+}$: 755.1709, found: 755.1706.

\section{Synthesis of compound 7}

$\mathrm{PhCH}=\mathrm{CH}_{2}(220 \mathrm{mg}, 2.12 \mathrm{mmol})$ was added to a solution of 1 $(1.00 \mathrm{~g}, 1.59 \mathrm{mmol})$ in hexane $(6 \mathrm{~mL})$ at room temperature, affording a white precipitate. After the reaction mixture was stirred for $15 \mathrm{~h}$, the supernatant was removed by filtration and the solid was dried under vacuum affording 7 as a white powder (890 mg, 76\%). Crystals suitable for X-ray diffraction were grown by slow diffusion of hexane into a solution of 7 in $\mathrm{CH}_{2} \mathrm{Cl}_{2} \cdot{ }^{1} \mathrm{H}$ NMR (400 MHz, $\left.\mathrm{C}_{6} \mathrm{D}_{6}\right): \delta[\mathrm{ppm}]=6.92-6.74(5 \mathrm{H}, \mathrm{Ph}), 6.42(\mathrm{~d}$, $2 \mathrm{H},{ }^{4} J_{\mathrm{P}-\mathrm{H}}=3 \mathrm{~Hz}$, meta-Mes), 6.32 (br s, 2H, meta-Mes), 4.76 (dt, $\left.1 \mathrm{H},{ }^{3} J_{\mathrm{H}-\mathrm{H}}=13 \mathrm{~Hz},{ }^{2} J_{\mathrm{P}-\mathrm{H}}=6 \mathrm{~Hz}, \mathrm{CH}\right), 2.96,1.95$ (each m, each $\left.1 \mathrm{H}, \mathrm{BCH} \mathrm{H}_{2}\right), 2.38-1.84\left(18 \mathrm{H}\right.$, Mes- $\left.\mathrm{CH}_{3}\right) .{ }^{13} \mathrm{C} \mathrm{NMR}(101 \mathrm{MHz}$, $\left.\mathrm{C}_{6} \mathrm{D}_{6}\right): \delta[\mathrm{ppm}]=148.1\left(\mathrm{dm},{ }^{1} J_{\mathrm{C}-\mathrm{F}}=241 \mathrm{~Hz}\right.$, ortho- $\left.C_{6} \mathrm{~F}_{5}\right), 139.7$ $\left(\mathrm{dm},{ }^{1} J_{\mathrm{C}-\mathrm{F}}=248 \mathrm{~Hz}\right.$, meta- $\left.C_{6} \mathrm{~F}_{5}\right), 137.5\left(\mathrm{dm},{ }^{1} J_{\mathrm{C}-\mathrm{F}}=249 \mathrm{~Hz}\right.$, para$C_{6} \mathrm{~F}_{5}$ ), 143.8, 143.4, 138.2 (ortho-Mes and para-Mes), 131.7, 131.4 (meta-Mes), 129.1, 128.5, $127.6(\mathrm{Ph}), 51.3\left(\mathrm{~d},{ }^{1} J_{\mathrm{P}-\mathrm{C}}=46 \mathrm{~Hz}\right.$, $\mathrm{PCH}), 35.6\left(\mathrm{BCH}_{2}\right), 23.5,22.4,20.7\left(\mathrm{Mes}-\mathrm{CH}_{3}\right) .{ }^{19} \mathrm{~F}$ NMR (376 $\left.\mathrm{MHz}, \mathrm{C}_{6} \mathrm{D}_{6}\right): \delta[\mathrm{ppm}]=-133.0\left(\mathrm{dm}\right.$, ortho $\left.-\mathrm{C}_{6} F_{5}\right),-159.4(\mathrm{t}$, para$\left.\mathrm{C}_{6} F_{5}\right),-164.4$ (meta, m-C $\left.{ }_{6} F_{5}\right) \cdot{ }^{31} \mathrm{P}$ NMR $\left(162 \mathrm{MHz}, \mathrm{C}_{6} \mathrm{D}_{6}\right): \delta[\mathrm{ppm}]$ $=82.4\left(\mathrm{~d},{ }^{2} J_{\mathrm{P}-\mathrm{H}}=39 \mathrm{~Hz}\right) .{ }^{11} \mathrm{~B} \mathrm{NMR}\left(160 \mathrm{MHz}, \mathrm{C}_{6} \mathrm{D}_{6}\right): \delta[\mathrm{ppm}]=$ 3.16. HRMS $(\mathrm{m} / \mathrm{z})$ : calcd for $\mathrm{C}_{38} \mathrm{H}_{30} \mathrm{OPBF}_{10} \mathrm{Na}[\mathrm{M}+\mathrm{Na}]^{+}$: 757.1865, found: 757.1865 .

\section{Synthesis of compound 8}

Isoprene (50 $\mathrm{mg}, 0.74 \mathrm{mmol}$ ) was added to a solution of 1 (331 $\mathrm{mg}, 0.525 \mathrm{mmol}$ ) in hexane $(3 \mathrm{~mL}$ ) at room temperature, affording a white precipitate after stirring for about $24 \mathrm{~h}$. The supernatant was removed by filtration and the solid was dried under vacuum affording 8 as a white powder (290 mg, 79\%). Crystals suitable for X-ray diffraction were grown by slow diffusion of hexane into a solution of 8 in $\mathrm{CH}_{2} \mathrm{Cl}_{2} \cdot{ }^{1} \mathrm{H} \mathrm{NMR}$ (400 $\left.\mathrm{MHz}, \mathrm{C}_{6} \mathrm{D}_{5} \mathrm{Br}\right): \delta[\mathrm{ppm}]=6.79(\mathrm{~s}, 2 \mathrm{H}$, meta-Mes $), 6.76\left(\mathrm{~d}, 2 \mathrm{H},{ }^{4} J_{\mathrm{P}-}\right.$ $\mathrm{H}=4 \mathrm{~Hz}$, meta-Mes), $5.16\left(\mathrm{~d}, 1 \mathrm{H},{ }^{2} J_{\mathrm{H}-\mathrm{H}}=3 \mathrm{~Hz}, \mathrm{C}=\mathrm{CH}_{2}\right), 5.03(\mathrm{~s}$, $\left.1 \mathrm{H}, \mathrm{C}=\mathrm{CH}_{2}\right), 4.58\left(\mathrm{dt}, 1 \mathrm{H},{ }^{3} J_{\mathrm{H}-\mathrm{H}}=12 \mathrm{~Hz},{ }^{2} J_{\mathrm{P}-\mathrm{H}}=7 \mathrm{~Hz}, \mathrm{CH}\right), 2.66$, 2.02 (each m, each $\left.1 \mathrm{H}, \mathrm{BCH}_{2}\right), 2.57-2.01\left(18 \mathrm{H}, \mathrm{Mes}^{-} \mathrm{CH}_{3}\right), 1.49(\mathrm{~s}$, $\left.3 \mathrm{H}, \mathrm{CH}_{3}\right) .{ }^{13} \mathrm{C}$ NMR (101 MHz, $\left.\mathrm{C}_{6} \mathrm{D}_{5} \mathrm{Br}\right): \delta[\mathrm{ppm}]=147.6(\mathrm{dm}$, ${ }^{1} J_{\mathrm{C}-\mathrm{F}}=241 \mathrm{~Hz}$, ortho- $\left.C_{6} \mathrm{~F}_{5}\right), 139.3\left(\mathrm{dm},{ }^{1} J_{\mathrm{C}-\mathrm{F}}=248 \mathrm{~Hz}\right.$, meta$\left.C_{6} \mathrm{~F}_{5}\right), 137.0\left(\mathrm{dm},{ }^{1} J_{\mathrm{C}-\mathrm{F}}=249 \mathrm{~Hz}\right.$, para- $\left.C_{6} \mathrm{~F}_{5}\right), 143.9$, 143.1, (orthoMes and para-Mes), $142.3\left(C=\mathrm{CH}_{2}\right), 131.8,131.3$ (meta-Mes), $116.9\left(\mathrm{~d},{ }^{3} J_{\mathrm{P}-\mathrm{C}}=12 \mathrm{~Hz}, \mathrm{C}=\mathrm{CH}_{2}\right), 52.1\left(\mathrm{~d},{ }^{1} J_{\mathrm{P}-\mathrm{C}}=46 \mathrm{~Hz}, \mathrm{PCH}\right)$, $29.9\left(\mathrm{BCH}_{2}\right), 23.4,23.0,21.1,20.9\left(\mathrm{Mes}-\mathrm{CH}_{3}\right), 19.9\left(\mathrm{CH}_{3}\right) .{ }^{19} \mathrm{~F}$ NMR (376 MHz, $\left.\mathrm{C}_{6} \mathrm{D}_{5} \mathrm{Br}\right): \delta-132.3\left(\mathrm{dm}\right.$, ortho- $\left.\mathrm{C}_{6} F_{5}\right),-158.9(\mathrm{dt}$, para- $\left.\mathrm{C}_{6} F_{5}\right),-163.6\left(\mathrm{~m}\right.$, meta- $\left.\mathrm{C}_{6} F_{5}\right) .{ }^{31} \mathrm{P}$ NMR $\left(162 \mathrm{MHz}, \mathrm{C}_{6} \mathrm{D}_{5} \mathrm{Br}\right)$ : $\delta[\mathrm{ppm}]=79.8\left(\mathrm{~d},{ }^{2} \mathrm{~J}_{\mathrm{P}-\mathrm{H}}=37 \mathrm{~Hz}\right) .{ }^{11} \mathrm{~B} \mathrm{NMR}\left(160 \mathrm{MHz}, \mathrm{C}_{6} \mathrm{D}_{5} \mathrm{Br}\right):$ $\delta[\mathrm{ppm}]=2.59$. HRMS $(\mathrm{m} / \mathrm{z})$ : calcd for $\mathrm{C}_{35} \mathrm{H}_{30} \mathrm{OPBF}_{10} \mathrm{Na}[\mathrm{M}+$ $\mathrm{Na}]^{+}:$721.1865, found: 721.1857 .

\section{Conflicts of interest}

There are no conflicts to declare.

\section{Acknowledgements}

Financial support from the National Natural Science Foundation of China $(21372048,21573044,21672039)$ and Shanghai Science and Technology Committee (16DZ2270100) is gratefully acknowledged. We also thank the High Performance Computer Center of Fudan University for the allocation of computing time. We thank Dr Yue-Jian Lin and Ms Haiwen Tian for help on crystallography study.

\section{Notes and references}

1 (a) G. C. Welch, R. R. S. Juan, J. D. Masuda and D. W. Stephan, Science, 2006, 314, 1124-1126; (b) D. W. Stephan and G. Erker, Angew. Chem., Int. Ed., 2010, 49, 46-76; (c) D. W. Stephan and G. Erker, Angew. Chem., Int. Ed., 2015, 54, 6400-6441; (d) D. W. Stephan, J. Am. 
Chem. Soc., 2015, 137, 10018-10032; (e) D. W. Stephan, Science, 2016, 354, aaf7229.

2 (a) T. A. Rokob, A. Hamza, A. Stirling, T. Soós and I. Pápai, Angew. Chem., Int. Ed., 2008, 47, 2435-2438; (b) Y. Guo and S. Li, Inorg. Chem., 2008, 47, 6212-6219; (c) T. A. Rokob, A. Hamza and I. Pápai, J. Am. Chem. Soc., 2009, 131, 1070110710; (d) S. Grimme, H. Kruse, L. Goerigk and G. Erker, Angew. Chem., Int. Ed., 2010, 49, 1402-1405; (e) T. A. Rokob, I. Bakó, A. Stirling, A. Hamza and I. Pápai, J. Am. Chem. Soc., 2013, 135, 4425-4437.

3 G. Kehr, S. Schwendemann and G. Erker, Top. Curr. Chem., 2012, 332, 45-84.

4 A. Schnurr, H. Vitze, M. Bolte, H.-W. Lerner and M. Wagner, Organometallics, 2010, 29, 6012-6019.

5 (a) F. Bertini, V. Lyaskovskyy, B. J. J. Timmer, F. J. J. Kanter, M. Lutz, A. W. Ehlers, J. C. Slootweg and K. Lammertsma, J. Am. Chem. Soc., 2012, 134, 201-204; (b) E. R. M. Habraken, L. C. Mens, M. Nieger, M. Lutz, A. W. Ehlers and J. C. Slootweg, Dalton Trans., 2017, 46, 12284-12292.

6 K. Samigullin, I. Georg, M. Bolte, H.-W. Lerner and M. Wagner, Chem.-Eur. J., 2016, 22, 3478-3484.

7 A. Stute, G. Kehr, R. Fröhlich and G. Erker, Chem. Commun., 2011, 47, 4288-4290.

8 (a) C. Appelt, H. Westenberg, F. Bertini, A. W. Ehlers, J. C. Slootweg, K. Lammertsma and W. Uhl, Angew. Chem., Int. Ed., 2011, 50, 3925-3928; (b) W. Uhl and C. Appelt, Organometallics, 2013, 32, 5008-5014; (c) W. Uhl, C. Appelt, A. Wollschläger, A. Hepp and E.-U. Würthwein, Inorg. Chem., 2014, 53, 8991-8999; (d) M. Devillard, E. Nicolas, A. W. Ehlers, J. Backs, S. Mallet-Ladeira, G. Bouhadir, J. C. Slootweg, W. Uhl and D. Bourissou, Chem. Commun., 2014, 50, 14805-14808; (e) M. Devillard, R. Declercq, E. Nicolas, A. W. Ehlers, J. Backs, N. Saffon-Merceron, G. Bouhadir, J. C. Slootweg, W. Uhl and D. Bourissou, J. Am. Chem. Soc., 2016, 138, 4917-4926; (f) L. Keweloh, H. Klöcker, E.-U. Würthwein and W. Uhl, Angew. Chem., Int. Ed., 2016, 55, 3212-3215.
9 C. Appelt, J. C. Slootweg, K. Lammertsma and W. Uhl, Angew. Chem., Int. Ed., 2012, 51, 5911-5914.

10 C. Appelt, J. C. Slootweg, K. Lammertsma and W. Uhl, Angew. Chem., Int. Ed., 2013, 52, 4256-4259.

11 J. Possart and W. Uhl, Organometallics, 2018, 37, 1314-1323. 12 (a) C. Rosorius, C. G. Daniliuc, R. Fröhlich, G. Kehr and G. Erker, J. Organomet. Chem., 2013, 744, 149-155; (b) Z. Jian, G. Kehr, C. G. Daniliuc, B. Wibbeling and G. Erker, Dalton Trans., 2017, 46, 11715-11721.

13 (a) X. Xu, R. Fröhlich, C. G. Daniliuc, G. Kehr and G. Erker, Chem. Commun., 2012, 48, 6109-6111; (b) X. Xu, G. Kehr, C. G. Daniliuc and G. Erker, J. Am. Chem. Soc., 2013, 135, 6465-6476.

14 A. W. Kyri, R. Kunzmann, G. Schnakenburg, Z.-W. Qu, S. Grimme and R. Streubel, Chem. Commun., 2016, 52, 13361-13364.

15 C. U. Grünanger and B. Breit, Angew. Chem., Int. Ed., 2008, 47, 7346-7349.

16 (a) Y. Zhao and D. G. Truhlar, J. Chem. Phys., 2006, 125, 194101; (b) Y. Zhao and D. G. Truhlar, J. Phys. Chem. A, 2006, 110, 5121-5129.

17 M. A. Dureen, C. C. Brown and D. W. Stephan, Organometallics, 2010, 29, 6594-6607.

18 (a) J. S. J. McCahill, G. C. Welch and D. W. Stephan, Angew. Chem., Int. Ed., 2007, 46, 4968-4971; (b) C. M. Mömming, S. Frömel, G. Kehr, R. Fröhlich, S. Grimme and G. Erker, J. Am. Chem. Soc., 2009, 131, 12280-12289.

19 M. Ullrich, K. S.-H. Seto, A. J. Lough and D. W. Stephan, Chem. Commun., 2009, 2335-2337.

20 J. M. Breunig, A. Hübner, M. Bolte, M. Wagner and H.-W. Lerner, Organometallics, 2013, 32, 6792-6799.

21 J. Ke, Y. Tang, H. Yi, Y. Li, Y. Cheng, C. Liu and A. Lei, Angew. Chem., Int. Ed., 2015, 54, 6604-6607.

22 D. J. Parks, W. E. Piers and G. P. A. Yap, Organometallics, 1998, 17, 5492-5503. 